\title{
New experimental tools for exploring in-medium parton propagation in QCD
}

\author{
William Brooks ${ }^{1}$, Hayk Hakobyan, and Miguel Arratia \\ Instituto para Estudios Avanzados en Ciencia y Tecnología \\ Centro Cientifico Tecnológico de Valparaíso \\ Universidad Técnica Federico Santa María, Valparaíso \\ Avda. España 1680, Casilla 110-V, Valparaíso, Chile \\ E-mail: william.brookseusm.cl
}

\begin{abstract}
Atomic nuclei can be employed as spatial analyzers of the propagation of partons in-medium and of the hadronization process. The study of semi-inclusive deep inelastic scattering on nuclei using fully-identified final state hadrons began with the HERMES program in the late 1990s, and is now continuing at Jefferson Lab. In the Jefferson Lab measurements, electrons and positive pions were measured from a $5 \mathrm{GeV}$ electron beam incident on targets of liquid deuterium, carbon, iron, and lead using CLAS in Hall B. The broadening of the transverse momentum of positive pions has been studied in detail as a function of multiple kinematic variables, and interpreted in terms of the transport of the struck quark through the nuclear systems. New insights are being obtained from these data concerning the roles of current and target fragmentation, characteristic time scales of the processes, and quantum interferences, in the interpretation of what is observed.
\end{abstract}

Another new probe of parton propagation through nuclear systems is provided by the protonlead collisions planned for the LHC later this year. The initial tests and plans for proton-lead collisions at the LHC will be described, and the physics that could be obtained from an extended $\mathrm{p}-\mathrm{Pb}$ run will be discussed; topics include gluon shadowing, nuclear parton distribution functions, and estimates of the saturation scale in cold nuclear matter.

These studies of the parton propagation and hadronization processes expose important connections between the DIS data, deuterium-gold collisions at RHIC, proton-nucleus interactions in Fermilab experiment E-906 at $120 \mathrm{GeV}$, and proton-nucleus collisions at the LHC with multi$\mathrm{TeV}$ beams. They are providing new tools for understanding the fundamental QCD processes at play. In addition, they may help to constrain the interpretation of the jet quenching seen in heavy ion collisions at the LHC and at RHIC. The DIS measurements will be extended in the next few years with the approved JLab experiment E12-06-117, and later will be pursued at the future Electron-Ion Collider.

Sixth International Conference on Quarks and Nuclear Physics, Ecole Polytechnique, Palaiseau, Paris

April 16-20, 2012

\footnotetext{
${ }^{1}$ Speaker
} 


\section{Introduction}

The propagation of partons through a strongly interacting medium is a ubiquitous phenomenon in all high-energy interactions involving nuclei. Its full description requires both perturbative and non-perturbative elements even at the highest energies, due to the close connections to the hadronization process and to soft interactions with the medium. The main ingredients considered for parton propagation through hot and cold matter are in-medium partonic multiple scattering, energy loss through gluon radiation and collisional losses, and the fragmentation of quarks and gluons to isolated hadrons and to jets, a process that can itself be modified by the medium. Modeling of hadronization began in the 1970's with the Lund string model, and in the 1980's the foundations of the complex monte carlo generators such as HERWIG and PYTHIA/JETSET were laid. Some theoretical effort was expended on understanding the space-time characteristics implied by the Lund model and the potential for exploiting DIS on nuclei [1], but experimental data with identified hadrons were not to become available for another decade until the advent of the HERMES data from HERA [2]. Meanwhile, interest in probing relativistic heavy ion collisions with hard probes grew and became a reality with the RHIC experimental that began in 1990, resulting in many detailed measurements and theoretical calculations. The principles for understanding in-medium energy loss in pQCD were established in the 1990's by BDMPS-Z [3], however, a number of new aspects of this process have been identified in the intervening years. These include estimates of the time dependence of the energy loss process for light quarks in vacuum [4], estimates for heavy quark mediumstimulated energy loss [5], and the development of new monte carlo simulations such as JEWEL [6] that are able to go beyond the limitations of analytic calculations. Recent progress on medium-modified fragmentation includes considerations of quantum-mechanical effects in cold nuclear matter [7], and most recently the impacts of the medium on color flow and correlations in multiple gluon radiation, such as the angular ordering within jets [8].

Figure 1 shows a schematic drawing of three processes which exemplify in-medium parton

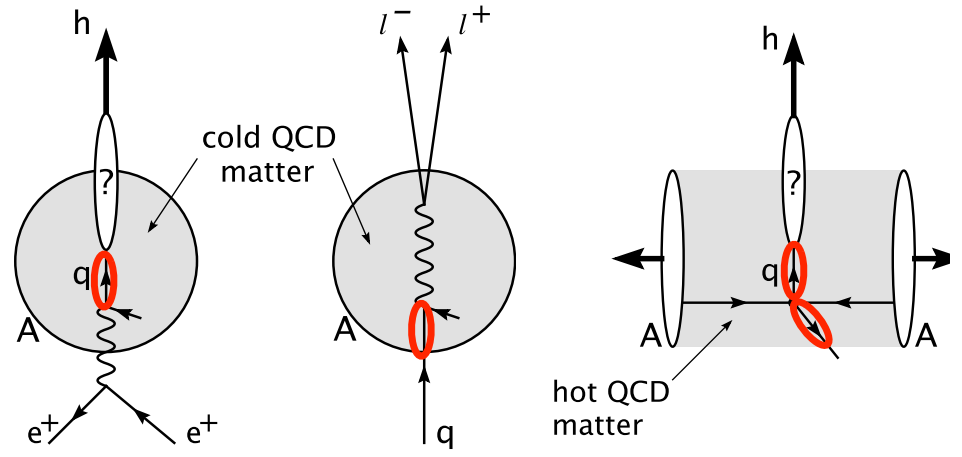

Figure 1 Parton propagation in semi-inclusive deep inelastic scattering for large $x$ (left), in the photon Drell-Yan reaction (center), and in relativistic heavy ion collisions (right). The red oval identifies the stage in which a parton is propagating through the strongly interacting medium. propagation. In the left drawing of this figure, semi-inclusive deep inelastic scattering (DIS) at large $\mathrm{x}$ is shown, where the virtual photon is absorbed by a single quark which propagates through the medium. Ultimately, a hadron is formed that contains the struck quark, accompanied by other hadrons. In the middle drawing of Fig. 1, the photon Drell-Yan (DY) process is shown, in which a parton from one hadronic system combines with a parton from the other to form an intermediate virtual 
photon that decays into a dilepton pair. In the right-hand drawing, two partons propagate through the hot dense medium formed by relativisitic heavy ion (RHI) collisions. In each drawing, a red oval marks the region where a parton is propagating through the medium. Besides the medium temperature, another difference in these three cases is that DIS has a finalstate interaction of the outgoing parton or hadron, while DY has an initial state interaction, and RHI collisions potentially have final-state interactions of multiple partons. Nonetheless, there are important elements in common among these, such as partonic transverse momentum broadening that can be characterized by the $\hat{q}$ transport parameter, longitudinal energy loss, and final-state jets and hadrons in DIS and RHI with spectra that evidence medium modification. In the following sections, new low-energy data for transverse momentum broadening of hadrons in DIS will be discussed, including a possible scheme for extracting a characteristic time, and a preliminary model evaluation of the observed pattern. Following that, a method is proposed to directly estimate quark energy loss at low energies. Finally, some highlights of proton-ion collisions at the LHC will be noted, probing cold QCD matter at unprecedently high energies.

\section{Transverse momentum broadening in semi-inclusive deep inelastic scattering}

The first study of $\mathrm{p}_{\mathrm{T}}$ broadening of identified hadrons in cold nuclear matter was published by the HERMES collaboration in 2010 [9]. This allowed the first comparison of the flavor dependence of the broadening on the final state hadron, comparing charged pions to each other and to $\mathrm{K}^{+}$, and provided the first experimental indication of the variable dependences.

Preliminary $\mathrm{p}_{\mathrm{T}}$ broadening data from the

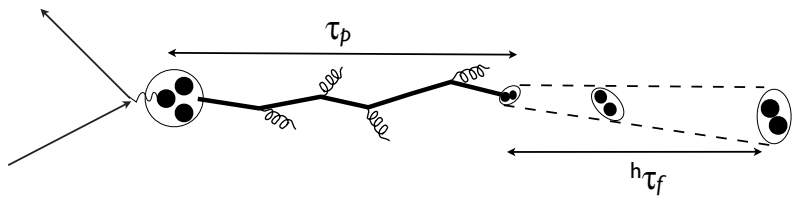

Figure 2 A schematic picture illustrating the definition of production time $\tau_{p}$ and formation time $\tau_{f}$ in deep inelastic production of hadrons. CLAS collaboration for charged and neutral pions are now becoming available as well, and with sufficient statistics to perform multi-dimensional binning to more completely probe the multi-variable dependences of this quantity, which is closely related to the transport parameter.

Figure 2 shows a schematic illustration of the trajectory of a parton following a deep inelastic scattering. The emission of gluons as indicated occurs in the vacuum process; in the medium there are additional medium-stimulated gluons emitted. These additional gluons, together with the smaller collisional losses, cause a broadening of the $\mathrm{p}_{\mathrm{T}}$ spectrum of the finalstate hadron that is formed. This broadening increases with the thickness of the nuclear medium, and thus can be studied by varying the nuclear target. Figure 3 shows $\mathrm{p}_{\mathrm{T}}$ broadening, defined as:

$$
\Delta \mathrm{p}_{\mathrm{T}}^{2} \equiv\left\langle\mathrm{p}_{\mathrm{T}}^{2}\right\rangle_{\mathrm{A}}-\left\langle\mathrm{p}_{\mathrm{T}}^{2}\right\rangle_{\mathrm{D}}
$$

for the new HERMES data together with CLAS preliminary data for positive pions, as well as pT broadening from the DY experiments E772 and E866 at Fermi National Accelerator Laboratory. The lines indicate the functional form $\mathrm{A}^{1 / 3}$ which is naively expected from a series of random, uncorrelated scatters. The CLAS data are shown in 27 bins of $\mathrm{Q}^{2}, v$ and $\mathrm{z}$ for $0.4<\mathrm{z}$ $<0.7$ and for carbon, iron, and lead targets.

As seen in Fig. 3, the CLAS data consistently fall below the line proportional to the average nuclear thickness, while the HERMES data are broadly consistent with proportionality. While a 
definitive explanation of this clear trend may require the more complete kinematic coverage that will be available following the $12 \mathrm{GeV}$ upgrade of JLab, a candidate explanation can be proposed based on the known features of this process. The most probable explanation in terms of a classical picture is that the prehadron production length, or color neutralization length, is shorter than the typical path length through the largest nucleus, $\mathrm{Pb}$. If this is the correct explanation, then it provides a means of measuring the production length with these data. This

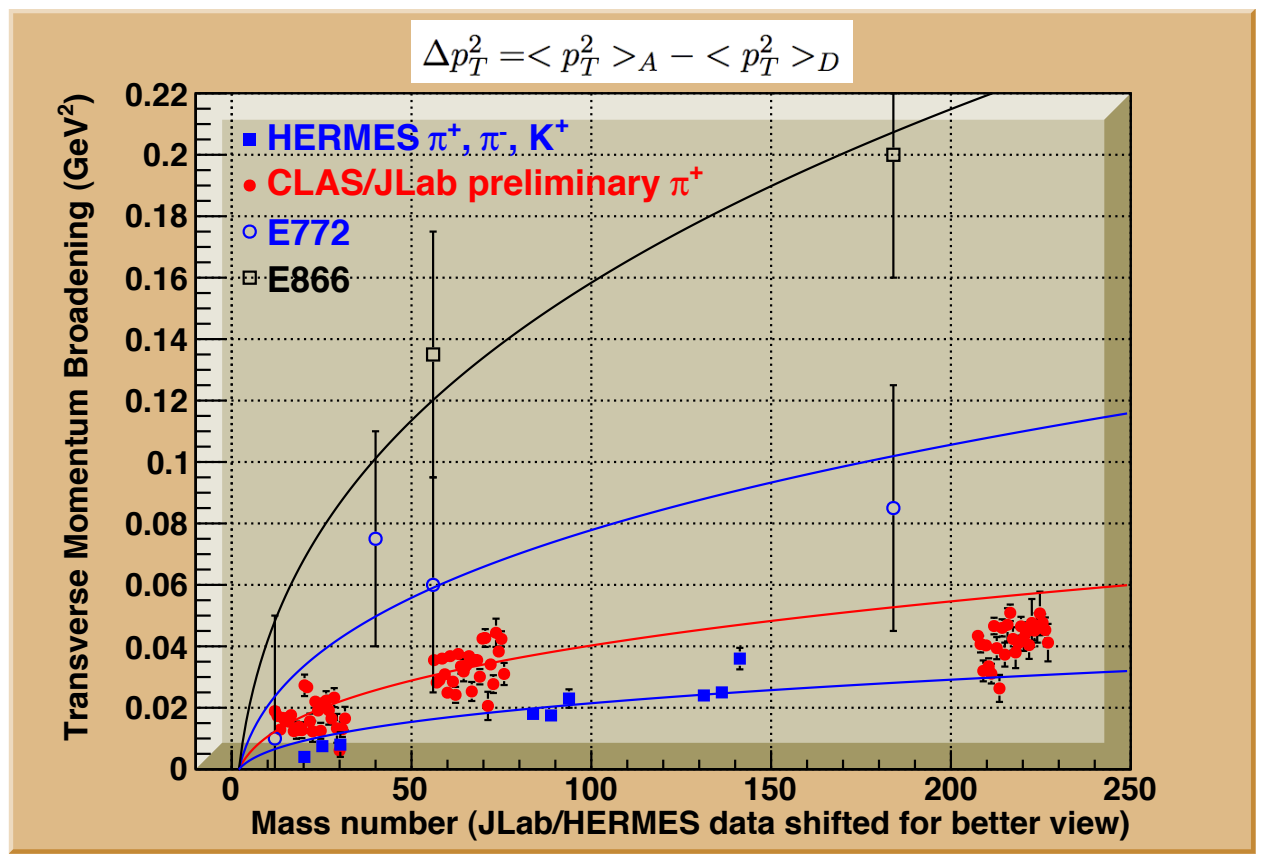

Figure 3 Transverse momentum broadening DIS data as a function of mass number. The data points are shown displaced to the right for visual clarity; the CLAS data are shown for mass 12, 56, and 207 for positive pions, while the HERMES data are shown for mass 20, 84, and 131 for charged pions and positive kaons. Data for E772 and E866 are for the Drell-Yan reaction.

concept is illustrated in Fig. 4, which shows the shape of the anticipated $\mathrm{p}_{\mathrm{T}}$ broadening curve vs $\mathrm{A}^{1 / 3}$ for different production lengths. If this is the correct explanation, it can ultimately be tested by checking the dependence on $v$ for fixed values of other variables: because the production length is due to a process taking place over a time interval, it is expected that time dilation will be visible through an increase of the production length at higher $v$. If this behavior is not observed, it would suggest that other mechanisms, e.g. coherence effects or second-order energy loss processes, are responsible for the non-proportionality of the broadening at the largest path lengths. Hints of quantum effects in $\mathrm{p}_{\mathrm{T}}$ broadening have been seen in the same data set and discussed previously, as well as the Feynman-x dependence of broadening [10].

In the hypothesis that the non-proportionality of the broadening is due to shorter production lengths, the CLAS data have been fitted by varying the production length in a classical geometric model that has a realistic nuclear density. Both the $\mathrm{p}_{\mathrm{T}}$ broadening and the hadron attenuation (multiplicity ratio) were fitted simultaneously. The resulting production lengths, defined with an exponential form, were in the range 1.6-2.0 fm. A plot from this model is shown in Fig. 5, giving the lengths within the medium for the partonic phase and the (pre)-hadronic phase for carbon, iron, and lead. Because the broadening in cold nuclear matter is small and has very little effect on the propagating parton, these studies probe space-time properties of the 
fundamental QCD vacuum process itself.

Another visible feature in Fig. 3 is that the magnitude of the HERMES broadening is smaller than that of the CLAS broadening. At least two effects contribute to this. First, there is a kinematic suppression of the hadronic broadening relative to the partonic broadening. It can be shown that to a very good approximation

$$
\Delta \mathrm{p}_{\mathrm{T}}^{2}=\mathrm{z}^{2} \Delta \mathrm{k}_{\mathrm{T}}^{2}
$$

where "p" refers to hadron momentum, " $\mathrm{k}$ " refers to parton momentum, and $\mathrm{z}$ is the usual relative energy $E_{h} / v$. The HERMES data are at a lower average value of $z$, and thus the observed broadening is smaller. The second potential effect is contribution of elastic scattering

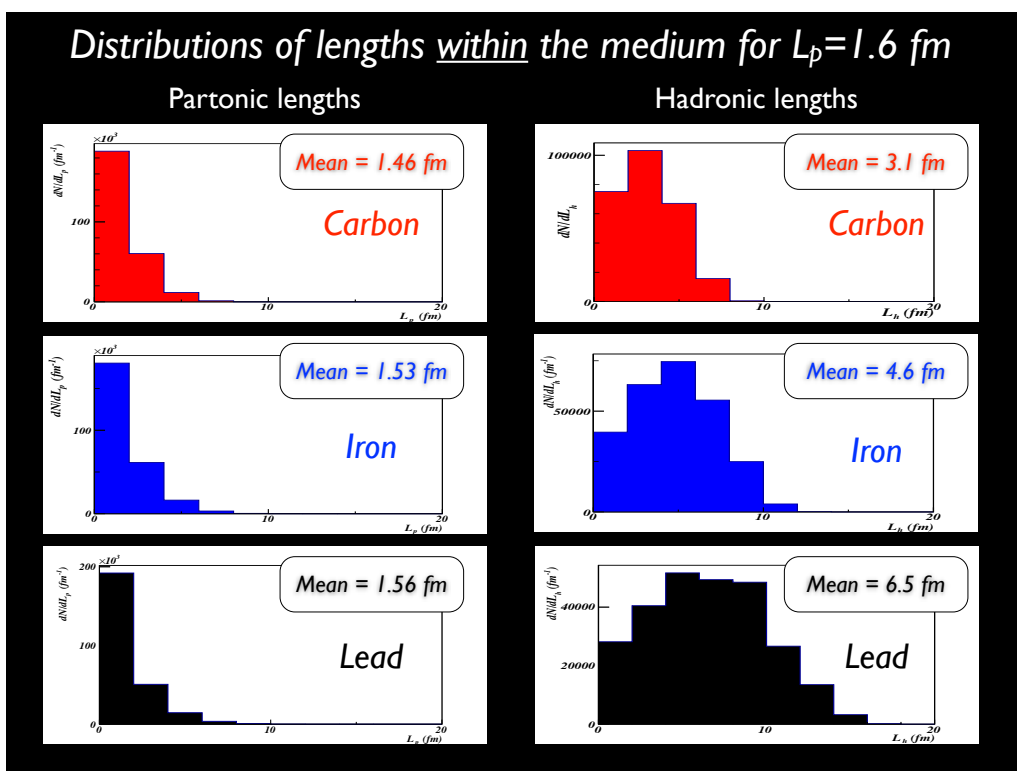

Figure 5 Distributions of path lengths within the medium. Result from a classical geometric model fitted simultaneously to the $p_{T}$ broadening and to the hadron attenuation from CLAS data for positive pions, plotted from 0 to $20 \mathrm{fm}$. The figures illustrate the expected behaviors due to edge effects and the boundaries of the nuclear density distributions.

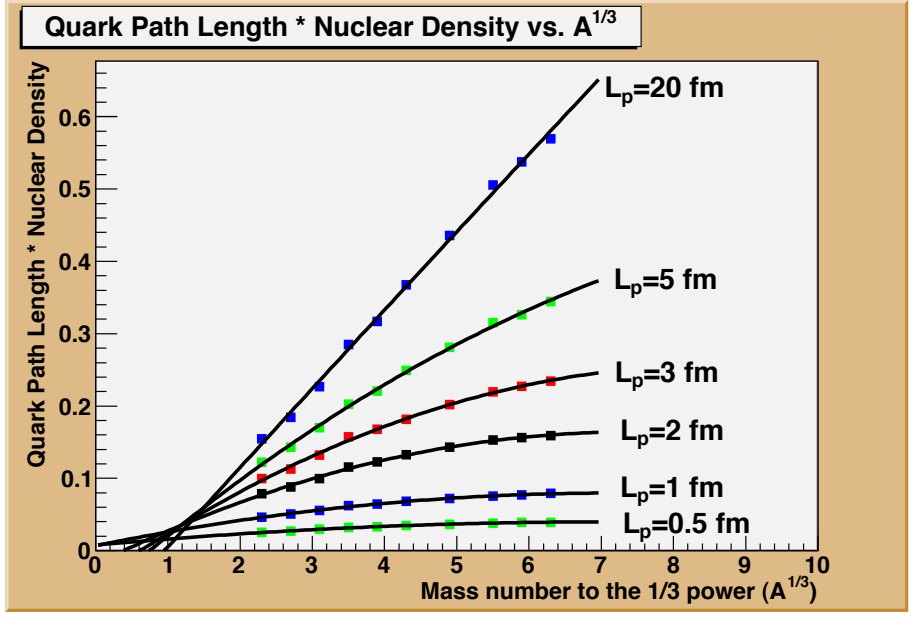

Figure 4 Monte Carlo calculation of the expected effect of the nuclear geometry on the $p_{T}$ broadening due to production lengths of varying magnitude. A realistic nuclear density distribution has been used. As expected, in a classical picture, for a production length much longer than the nuclear radius, broadening proportional to the average path length is seen. For shorter production lengths, the broadening is reduced, and a curvature develops. This behavior can be used to estimate the production length from experimental data.

of the pre-hadron or hadron to the observed broadening, which is expected to be more prominent at the lower energies. This has been studied in CLAS, and the data suggest that this is a small effect that is only visible for $\mathrm{z}>0.8$ for the larger nuclei. However, more study is needed, and the ultimate determination of this contribution will be fully realized with the upgraded CLAS12 detector and $11 \mathrm{GeV}$ beam. 


\section{Direct measurement of quark energy loss in semi-inclusive deep inelastic scattering}

Partonic energy loss has proven to be elusive to quantify experimentally. Experiments in cold nuclear matter with high energy beams have yielded some estimates but confounding effects such as nuclear shadowing have created ambiguity in the interpretations. Partonic energy loss has been studied at great length in heavy ion collisions but the emphasis is on using energy loss as a diagnostic tool rather than as a direct object of study, since variables such as temperature and density are not fixed, and the spatial and temporal evolution of the system is not yet uniquely determined. The role of DIS at low energies and $\mathrm{x}_{\mathrm{Bj}}>0.1$ on nuclear targets could be important in testing and refining the current understanding of partonic energy loss, since the initial energy of the propagating quark is completely known from the electron kinematics. At relatively low energies, the final state hadron energy also is moderate, so the effect of any energy loss must be more visible than at higher energies.

As a numerical example, recall the basic pQCD formula that relates radiative energy loss and $\mathrm{p}_{\mathrm{T}}$ broadening of partons (e.g., from reference [3]):

$$
-\frac{d E}{d x}=\frac{\alpha_{s} N_{c}}{4} \Delta k_{T}^{2}
$$

Taking $0.04 \mathrm{GeV}^{2}$ as an estimate of the pion broadening for Pb from the CLAS data of Fig. 3, and dividing by $\mathrm{z}^{2}$ for $\mathrm{z}=0.5$ as discussed at the end of the previous section, one arrives at a quark-level broadening of $0.16 \mathrm{GeV}^{2}$. For $\alpha_{\mathrm{s}} \sim 0.3$, this gives $\mathrm{dE} / \mathrm{dx} \sim 150 \mathrm{MeV} / \mathrm{fm}$. For the expected quadratic energy loss, at the radius of $\mathrm{Pb}$ this yields a total average energy loss of 600 $\mathrm{MeV}$. For pions in the few-GeV energy range, this is a very large energy loss that should be visible by inspection of the spectra. However, there are several other considerations. For example, the above formulas were developed with a number of assumptions, such as the eikonal approximation. There could also be non-perturbative ingredients. Finally, effects such as the time required for initiation and equilibration of medium induced energy loss have been neglected, and this can be a substantial fraction of the total time the parton spends in-medium. Thus, there is motivation to gather as much experimental information as possible to test and refine our understanding of the fundamental processes involved.

Toward this end, we are testing methods to try to 'directly' measure quark energy loss in high-x DIS by comparing the energy of positive pions emerging from the current fragmentation region in electron-deuteron scattering to those from electron-lead scattering. We focus on the energy spectrum of pions so as to directly extract energy loss, rather than looking at the $\mathrm{z}$ spectrum. In the simplest possible case, one could hope for a 'simple shift' of the pion energy spectrum resulting from the energy loss. However, there are several factors that complicate this picture. First, the data is an average over the nuclear geometry, with the hard scattering occurring through the nuclear volume. Second, and more difficult, the quark fragments with a parton fragmentation function that further convolutes the shift to lower energies, diluting the effect, although similarly for the two targets. Qualitatively, it appears on inspection that the energy loss is substantially smaller than the $600 \mathrm{MeV}$ estimate. As seen in Fig. 6, following the $\mathrm{X}_{\mathrm{F}}$ cut, the normalized $\pi^{+}$energy spectrum shape looks very similar for deuterium and $\mathrm{Pb} . \mathrm{A}$ small shift of the higher-energy part of the spectrum is visible, consistent with a few tens of $\mathrm{MeV}$ of energy loss. Different normalization conventions can produce different estimates, and further study is needed, including modeling with simultaneous comparison to both the $\mathrm{z}$ spectrum and energy spectrum. Nonetheless, it seems that average shifts at the level of 600 


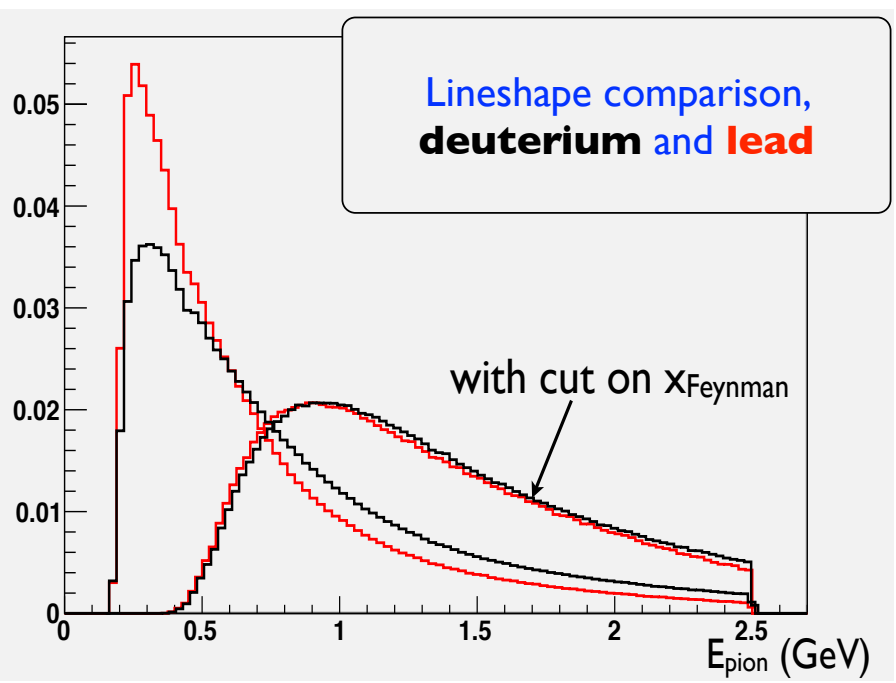

Figure 6 Lineshape comparison of DIS positive pion energies normalized to unity, with and without a requirement on Feynman $x\left(x_{F}>0.1\right)$. Comparison of the two distributions with the $x_{F}$ cut may allow a direct estimate of quark energy loss. Qualitatively, the energy loss appears to be substantially smaller than that given by the perturbative formula (see text). The cutoff at $2.5 \mathrm{GeV}$ is due to the limitations of the particle ID system.

superposition of elementary interactions. These include an unexplained strong enhancement of proton production in central collisions, a strong enhancement of $\pi^{0}$ events in peripheral collisions with no suppression in central collisions, an enhancement of jets in peripheral collisions and suppression in central collisions, a strong enhancement of electrons from heavy flavor decays in central collisions, and more [11]. Will these puzzling effects be seen at the LHC, with 25 times larger CM energies?

An example of a relevant connection between the low energy studies and high energy studies is the question of whether quark hadronization is fundamentally affected by the medium or not, independent of the distance over which color neutralization takes place. The high energies rule out the formation of a pre-hadron in the medium, but the color lines resulting from the rapid evolution in parton virtuality still pass through the medium and can be modified, altering the properties of the hadrons in the final state. Other parton propagation topics include saturation of partonic densities at low $\mathrm{x}$ and modification of the photon Drell-Yan process.

\section{Conclusions}

New experimental tools for exploring topics of in-medium parton propagation have been discussed; these have relevance for experiments with nuclei at the $\mathrm{GeV}$ scale and above. Transverse momentum broadening in semi-inclusive deep inelastic scattering at low energies can be used as a probe of fundamental QCD processes. New insights are being obtained from these data concerning the roles of current and target fragmentation, characteristic time scales of the processes, and quantum interferences, as well as the possibility that quark energy loss inmedium can be understood more deeply. At highest energies, proton-lead collisions will shed light on related ideas in a different kinematic regime, clarifying such questions as how 
hadronization is modified in-medium. These studies elucidate fundamental processes in QCD as well as providing constraints on the interpretation of phenomena observed in heavy ion collisions.

\section{Acknowledgements}

The authors gratefully acknowledge generous support from Chilean FONDECYT grants 1120953 and 3100064, Chilean CONICYT grant ACT-119, and Chilean BASAL grant FB0821.

\section{References}

[1] "Leptoproduction of hadrons from nuclear targets and fragmentation of quarks into hadrons," A. Bialas and T. Chmaj, Phys. Lett. 133B, 241 (1983); "Lund model and an outside-inside aspect of the inside-outside cascade,” A. Bialas and M. Gyulassy, Nucl. Phys. B291, 793 (1987).

[2] "Multidimensional Study of Hadronization in Nuclei," A. Airapetian et al. (HERME Coll.), Eur. Phys. J. A47:113 (2011) and references therein.

[3] See references in recent reviews: "Energy losses in a hot plasma revisited," S. Peigné and A. V. Smilga, Phys. Usp. 52:659-685, 2009 and Usp. Fiz. Nauk 179:697-726, 2009; "Parton Propagation and Fragmentation in QCD Matter," A. Accardi, F. Arleo, W. K. Brooks, D. D'Enterria, V. Muccifora, Rivista del Nuovo Cimento, Issue 09-10, 439-553 (2009); and "The theory and phenomenology of perturbative QCD based jet quenching," A. Majumder and M. Van Leeuwen, Prog. Part. Nucl. Phys 66 (2011) 41-92.

[4] "Why heavy and light quarks radiate energy with similar rates," B. Z. Kopeliovich, I. K. Potashnikova, and Iván Schmidt, Phys. Rev. C82 (2010) 037901.

[5] "Multiple parton scattering in nuclei: heavy quark energy loss and modified fragmentation functions," B.-W. Zhang, E. Wang, X.-N. Wang, Nuclear Physics A 757 (2005) 493-524; and [3].

[6] “JEWEL - a Monte Carlo Model for Jet Quenching," K. Zapp, J. Stachel, and U. A. Wiedemann, PoS High-pTphysics09 2009:022, 2009.

[7] "Quantum-mechanical description of in-medium fragmentation," B. Z. Kopeliovich, H.-J. Pirner, I. K. Potashnikova, Ivan Schmidt, A. V. Tarasov, O. O. Voskresenskaya, Phys. Rev. C78:055204 (2008).

[8] "The contribution of medium-modified color flow to jet quenching," A. Beraudo, J.G. Milhano, U.A. Wiedemann, JHEP 1207 (2012) 144, and references therein; "The radiation pattern of a QCD antenna in a dilute medium," Yacine Mehtar-Tani, Carlos A. Salgado, Konrad Tywoniuk, 10.1007/ JHEP04 (2012) 064; and references therein.

[9] "Transverse momentum broadening of hadrons produced in semi-inclusive deep-inelastic scattering on nuclei," A. Airapetian et al. (HERMES Coll.), Phys. Lett. B684:114-118, 2010

[10] "Studies of hadronization mechanisms using pion electroproduction in deep inelastic scattering from nuclei," W. K. Brooks, H. Hakobyan, C. Peña, M. Arratia, AIP Conf. Proc. 1441, 211 (2012).

[11] "PHENIX Results on Cold Nuclear Matter," M. Wysocki on behalf of the PHENIX Coll., Quark Matter 2012 Conference, August 2012 [ https://indico.cern.ch/getFile.py/access? contribId $=628 \&$ sessionId $=1 \&$ resId $=0 \&$ materialI $=$ =slides $\&$ confId $=181055]$ ] 